Pacific Journal of Mathematics

LOCALLY COMPLETE GRAPHS 


\section{LOCALLY COMPLETE GRAPHS}

HeNRy SHARP, JR.

If $G$ is the square of a graph $H$, then each vertex has a closed neighborhood which generates a complete subgraph of $G$ and $G$ is the union of these complete subgraphs. Although the converse fails, it does suggest a classification which yields a theory extensive enough to be of independent interest. This paper develops some basic properties of what will be called locally complete graphs. In $\S 3$ the theory is applied to the problem of square roots, and an existence theorem is proved from which Mukhopadhyay's theorem [3] follows as a corollary. Based on the more general theorem, a technique for square root determination is illustrated in the final section.

1. Introduction. A graph is a finite set of vertices together with some of its doubleton subsets, called edges. The closed neighborhood of a vertex $v$ is the set of all vertices at a distance not greater than 1 from $v$. If $V$ denotes a subset of the vertex set of a graph $G$, then the maximal subgraph of $G$ on the vertex set $V$ is said to be generated by $V$. Throughout this paper, $G$ will represent a nontrivial, connected graph. Terminology is essentially that of [2], in which all basic definitions may be found.

Definition 1. Let $\left\{v_{1}, v_{2}, \cdots, v_{n}\right\}$ be the vertex set of a graph $G$, and for each $\alpha$ let $N_{\alpha}^{*}$ denote the closed neighborhood of $v_{\alpha}$. Let $N_{\alpha}$ be any subset of $N_{\alpha}^{*}$ containing $v_{\alpha}$ which generates a complete subgraph $C_{\alpha}$ of $G$. Then $C_{\alpha}$ is called a complete subneighborhood of $v_{\alpha}$, and the indexed family $\mathscr{C}=\left\{C_{1}, C_{2}, \cdots, C_{n}\right\}$ is called a complete family for $G$ if $G=\bigcup \mathscr{C}$. A graph $G$ is called locally complete iff $G$ has at least one complete family.

It is easily seen that complete graphs, trees, and unicyclic graphs are also locally complete. The complete bigraph $K_{3,2}$ is the smallest (nontrivial, connected) graph which fails to be locally complete. The following lemma, suggested by $K_{3,2}$, depends upon the observation that if the given graph contains more than one cycle then the number of edges exceeds the number of vertices.

Lemma. A graph $G$ which contains no triangle is locally complete iff it contains at most one cycle.

No full characterization of local completeness is known. An 
additional result along this line, however, is given in the theorem below.

THEOREM 1. Let the graph $G$ contain a clique $T$ of order $r>2$ and suppose that $T$ has no edge in common with any other clique in $G$. Then $G$ is locally complete iff for some set $E$ of $\left(\begin{array}{l}r \\ 2\end{array}\right)-1$ edges in $T$ each nontrivial component of $G-E$ is locally complete.

Proof. Let $G$ be locally complete. Some vertex in $T$ has a subneighborhood contained in $T$, hence there exists a complete family $\mathscr{C}$ in $G$ which contains $T$. Let $v_{\beta}$ denote a vertex in $T$ such that $C_{\beta}=T$. Let $e_{\beta}$ be any edge in $T$ incident with $v_{\beta}$, and let $E$ be the set of all edges in $T$ except $e_{\beta}$. For each $\alpha$ let $C_{\alpha}^{\prime}=C_{\alpha} \cap(G-E)$. If $C_{\alpha}^{\prime}$ contains an edge $\neq e_{\beta}$ then $C_{\alpha}$ does not contain an edge of $T$. Therefore $C_{\alpha}^{\prime}=C_{\alpha}$ and we put $C_{\alpha}^{\prime \prime}=C_{\alpha}^{\prime}=C_{\alpha}$. If $C_{\alpha}^{\prime}$ does not contain any edge and if $v_{\alpha}$ is not an isolated point in $G-E$, then define $C_{\alpha}^{\prime \prime}$ to be any complete subgraph of $G-E$ which contains $v_{\alpha}$. We need not be concerned with isolated vertices, and we note that $C_{\beta}^{\prime \prime}$ is defined to be $e_{\beta}$ plus its end points.

Now let $H$ be any nontrivial component of $G-E$, and put $K=$ $\bigcup_{v_{\alpha} \in H} C_{\alpha}^{\prime \prime}$. Each $C_{\alpha}^{\prime \prime}$ is connected, hence $K \subset H$. If $e_{\beta} \in H$ then $v_{\beta} \in H$ and $e_{\beta} \in K$. For any other edge $e \in H$, there is a $\gamma$ such that $e \in C_{r}=C_{r}^{\prime \prime} \subset K$. Therefore, $K=H$ and $H$ is locally complete.

Conversely suppose that there is a set $E$ of $\left(\begin{array}{l}r \\ 2\end{array}\right)-1$ edges in $T$ such that each nontrivial component of $G-E$ is locally complete. Note that if $v_{\alpha} \notin T$ then $v_{\alpha}$ is in a nontrivial component of $G-E$. For $v_{\alpha}$ is not isolated in $G$ and no edge of $G$ incident with $v_{\alpha}$ is in $T$. Let $C_{\delta}^{\prime}$ be the complete subneighborhood in $G-E$ for each nonisolated vertex $v_{\hat{\delta}}$.

Now let $\left(v_{\beta} v_{\gamma}\right)$ be the edge of $T$ in $G-E$ and suppose it is in a component $H$ of $G-E$. Then $\left(v_{\beta} v_{\gamma}\right)$ is not in a subgraph of $H$ isomorphic to $K_{m}$ for any $m>2$. If $\left\{C_{x x}^{\prime}\right\}$ is the complete family for $H$, then $\left(v_{\beta} v_{\gamma}\right) \in C_{\beta}^{\prime}$ or $C_{\gamma}^{\prime}$ and is the only edge in that subgraph. For definiteness, assume that $C_{\beta}^{\prime}=[\beta, \gamma]^{1}$. Then the following is a complete family $\mathscr{C}$ in $G$ :

$$
\begin{aligned}
& C_{\beta}=T, \\
& C_{\alpha}=T \text { if } v_{\alpha} \text { is isolated in } G-E, \\
& C_{\delta}=C_{\delta}^{\prime} \text { for all other vertices. }
\end{aligned}
$$

This theorem and the preceding lemma enable us to characterize all those graphs containing exactly one triangle.

1 We use $[\alpha, \beta, \cdots, \delta]$ to denote the complete graph on the vertices $v_{\alpha}, v_{\beta}, \cdots, v_{\delta}$. 
COROLLARY. If $G$ contains exactly one triangle $T$, then $G$ is locally complete iff for some pair, $E$, of edges in $T$ each nontrivial component of $G-E$ contains at most one cycle.

2. Derived graphs. The definition of complete family admits the possibility that some $C_{\alpha}$ may be trivial $\left(C_{\alpha}=\left\{v_{\alpha}\right\}\right)$ and that nontrivial complete subneighborhoods may have edges in common.

Definition 2. Two complete subneighborhoods are edge disjoint iff they have no edge in common. A complete family is edge disjoint iff its members are pairwise edge disjoint.

A locally complete graph may have more than one complete family $\mathscr{C}$ associated with it, and some of these may be edge disjoint. But not every locally complete graph has an edge disjoint complete family. The graph $G_{1}$ in Figure 1, for example, has no edge disjoint complete family, for if it did then at most one $C_{\alpha}$ could be a triangle,

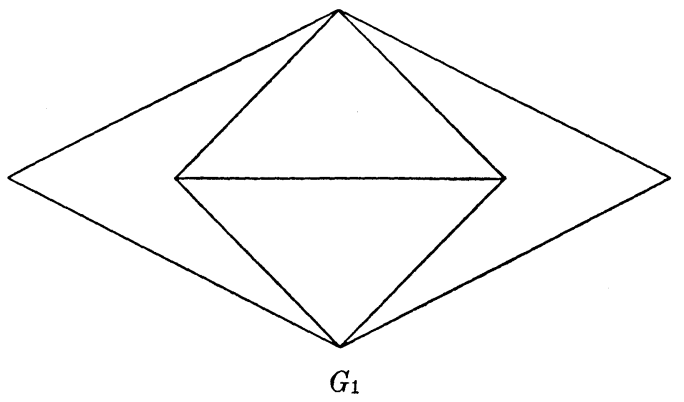

FIGURE 1

leaving five vertices but six edges. That $G_{1}$ is the smallest such graph can be seen in the following way. Let $\mathscr{C}$ be a complete family for any graph $G$. If $C_{\alpha} \subset C_{\beta}$ for some $\beta \neq \alpha$ then $C_{\alpha}$ can be redefined as $C_{\alpha}^{\prime}=\left\{v_{\alpha}\right\}$ thus insuring that $C_{\alpha}^{\prime}$ and $C_{\beta}$ are edge disjoint.

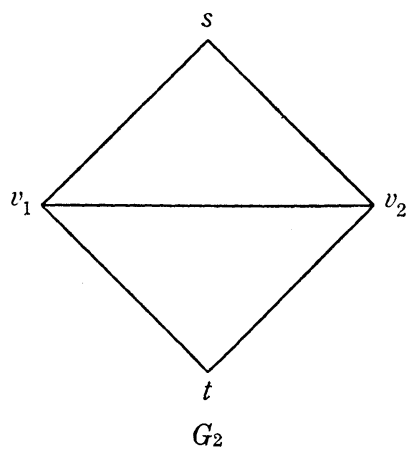

$s \in C_{\alpha}-C_{\beta}$

$t \in C_{\beta}-C_{\alpha}$

FIGURE 2 
If $G$ is locally complete and has no edge disjoint complete family then there is an edge $\left(v_{1} v_{2}\right) \in C_{\alpha} \cap C_{\beta}$, where neither complete subneighborhood is contained in the other and each contains at least three vertices. Thus $G$ contains a subgraph isomorphic to $G_{2}$ in Figure 2. It is easy to see that $G_{2}$ has an edge disjoint complete family. Furthermore, each locally complete graph with five vertices having $G_{2}$ as a subgraph also has an edge disjoint complete family.

Definition 3. Let $G$ be a locally complete graph and let $\mathscr{C}$ be a complete family for $G$. Corresponding to $\mathscr{C}$ there is a subgraph of $G$ called the derived graph $G(\mathscr{C})$ constructed as follows:

(1) $G(\mathscr{C})$ has the same vertex set as $G$,

(2) $\left(v_{\alpha} v_{\beta}\right) \in G(\mathscr{C})$ iff $\left(v_{\alpha} v_{\beta}\right) \in C_{\alpha} \cap C_{\beta}$.

The extreme case in which $G(\mathscr{C})$ is connected is that in which $G(\mathscr{C})=G$ and, as one might suspect, this case occurs iff $G$ is complete and for each $\alpha, C_{\alpha}=G$. At the opposite extreme, $G(\mathscr{C})$ is totally disconnected if $\mathscr{C}$ is edge disjoint, but the converse fails as may be observed in $G_{1}$. Disconnectedness of $G(\mathscr{C})$ depends upon properties of $G$ as well as upon the complete family $\mathscr{C}$. For example, if $G$ is locally complete and has a cut point, then $G(\mathscr{C})$ is not connected. This observation is generalized in the following theorem.

Theorem 2. Let $G$ be locally complete, let $V$ denote its vertex set and let $\mathscr{C}$ be a complete family for $G$. If $U=\left\{v_{1}, v_{2}, \cdots, v_{k}\right\}$ is any subset of $V$ let $U(\mathscr{C})=\left\{v \in V: v \in\left(C_{1} \cup C_{2} \cup \cdots \cup C_{k}\right)\right\}$, and $\bar{U}=$ $U(\mathscr{C})-U$. Then $G(\mathscr{C})$ is not connected if there is a nonempty proper subset $U$ of $V$ such that

$$
U \cap \bar{U}(\mathscr{C})=\varnothing
$$

Proof. Note that $\bar{U}$ is the set of vertices in the complement of $U$, each belonging to a complete subneighborhood of some vertex in $U$. Now consider the complete subneighborboods corresponding to vertices in $\bar{U}$. There can be no edge in $G(\mathscr{C})$ joining $U$ to its complement unless $U \cap \bar{U}(\mathscr{C}) \neq \varnothing$.

In spite of its easy proof, Theorem 2 has a practical value which will appear in the next section: to test whether $U$ and $V-U$ form a disconnection in $G(\mathscr{C})$ it is necessary to look only at vertices in $\bar{U}$, not at all vertices in $V-U$.

COROLLARY. If $\mathscr{C}$ is a complete family for $G$, then $G(\mathscr{C})$ is not connected if either

(a) $G$ has a vertex $v_{\alpha}$ such that $v_{\alpha} \notin \bigcup \bigcup\left\{C_{\beta}: v_{\beta} \in C_{\alpha}-v_{\alpha}\right\}$, or 
(b) $G$ has more that 2 vertices and has an edge not contained in a triangle.

A locally complete graph may contain several derived graphs and to study the relationship between them, it will be convenient to separate the edges of $G$ into the following types with respect to a given complete family:

( I ) $\left(v_{\alpha} v_{\beta}\right) \in C_{\alpha} \cap C_{\beta}$,

(II) $\left(v_{\alpha} v_{\beta}\right) \notin C_{\alpha} \cup C_{\beta}$,

(III) $\left(v_{\alpha} v_{\beta}\right)$ is in exactly one of $C_{\alpha}, C_{\beta}$.

Let $\mathscr{C}$ and $\mathscr{C}^{\prime}$ denote complete families for the graph $G$. It follows immediately that if $C_{\alpha} \subset C_{\alpha}^{\prime}$ for each $\alpha$ then $G(\mathscr{C}) \subset G\left(\mathscr{C}^{\prime}\right)$. Simple examples can be constructed to show that the converse fails; however, the next theorem provides a partial converse.

TheOREM 3. Let $\mathscr{C}$ and $\mathscr{C}^{\prime}$ denote complete families for the graph $G$.

(1) If $G$ has no edge of Type III with respect to $\mathscr{C}$, then $G(\mathscr{C}) \subset G\left(\mathscr{C}^{\prime}\right)$ implies $C_{\alpha} \subset C_{\alpha}^{\prime}$ for each $\alpha$.

(2) If $G$ has no edge of Type III with respect to either $\mathscr{C}$ or $\mathscr{C}^{\prime}$, then $\mathscr{C} \neq \mathscr{C}^{\prime}$ implies $G(\mathscr{C}) \neq G\left(\mathscr{C}^{\prime}\right)$.

The proof of this theorem offers no difficulty and is omitted.

If $\mathscr{L}$ is the set of all complete families $\mathscr{C}$ for $G$, then $\bigcup\{G(\mathscr{C}): \mathscr{C} \in \mathscr{L}\} \subset G$. The inclusion may be proper, as illustrated by the $(5,5)$ graph consisting of a 4-cycle plus an edge with one vertex on the cycle and one off. It would be of interest to determine conditions under which a locally complete graph is the union of its derived subgraphs. The (apparently) more complicated problem of reconstructing $G$ from its derived subgraphs has not yet been studied.

3. Squares and square roots. We now apply the ideas developed in the preceding sections to the problem of the existence and construction of square roots.

Let $H$ be a nontrivial, connected graph, let $N_{\alpha}^{*}$ be the closed neighborhood of $v_{\alpha}$ in $H$, and let $C_{\alpha}^{*}$ be the complete graph on $N_{\alpha}^{*}$. Although $C_{\alpha}^{*}$ need not be a subgraph of $H$, it is a subgraph of $H^{2}=$ $G$, and a simple argument shows that $G$ is the union of these complete subgraphs. Thus $G$ is locally complete, and we shall say that the complete family $\mathscr{C}^{*}=\left\{C_{1}^{*}, C_{2}^{*}, \cdots, C_{n}^{*}\right\}$ for $G$ is induced by $H$.

We note first that $G$ has no edge of Type III with respect to $\mathscr{C}^{*}$ and that $H=G\left(\mathscr{C}^{*}\right)$. Thus from Theorem 3 in the preceding section, if $\mathscr{C}$ is any complete family for $G$, then $H \subset G(\mathscr{C})$ iff 
$C_{\alpha}^{*} \subset C_{\alpha}$ for each $\alpha$. Although each square root is a derived graph, a derived graph, in general, need not be a square root. The graph $G_{3}$ in Figure 3, for example, is the complete graph $K_{4}$ and has a square root (indeed, its derived graph with respect to $\mathscr{C}$ is connected), yet $(G(\mathscr{C}))^{2} \neq G$. On the other hand, it is always true that $(G(\mathscr{C}))^{2} \subset G$.

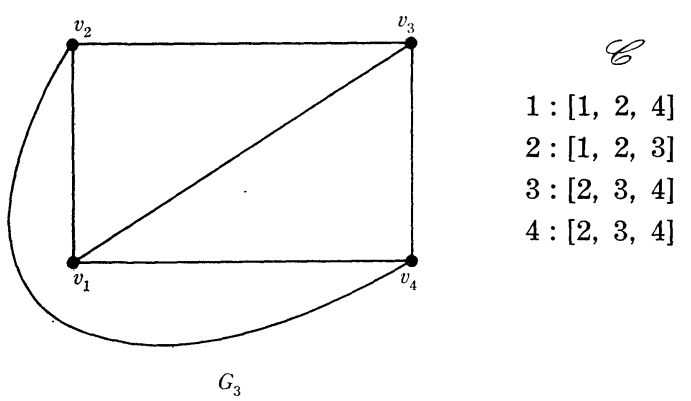

FIGURE 3

Summarizing these remarks, if $G$ has a square root, then it is locally complete and each of its square roots is a derived graph. But a locally complete graph $G$ may have derived graphs which are not square roots; in fact, each of its derived graphs may fail to be a square root. The next theorem characterizes those graphs which do have square roots.

THEOREM 4. Let $G$ be locally complete, with complete family $\mathscr{C}$. Then $(G(\mathscr{C}))^{2}=G$ iff for each $\left(v_{\alpha} v_{\beta}\right) \in G$ there is an index $\gamma$ such that (1) $v_{r} \in C_{\alpha} \cap C_{\beta}$ and (2) $\left(v_{\alpha} v_{\beta}\right) \in C_{\gamma}$.

Proof. Since $(G(\mathscr{C}))^{2} \subset G$ we need to show that the given condition is equivalent to $G \subset(G(\mathscr{C}))^{2}$.

First suppose that $G \subset(G(\mathscr{C}))^{2}$ and that $\left(v_{\alpha} v_{\beta}\right) \in G$. Then either $\left(v_{\alpha} v_{\beta}\right) \in G(\mathscr{C})$ or for some $\gamma\left(v_{\gamma} v_{\alpha}\right) \in G(\mathscr{C})$ and $\left(v_{\gamma} v_{\beta}\right) \in G(\mathscr{C})$. In the first case, conditions (1) and (2) hold with $\gamma=\alpha$ or $\gamma=\beta$. In the second case, $\left(v_{\gamma} v_{\alpha}\right) \in C_{\gamma} \cap C_{\alpha}$ and $\left(v_{\gamma} v_{\beta}\right) \in C_{\gamma} \cap C_{\beta}$, so that (1) and (2) hold.

Next suppose that conditions (1) and (2) hold and that $\left(v_{\alpha} v_{\beta}\right) \in G$. Then for some $\gamma,\left(v_{\gamma} v_{\alpha}\right) \in C_{\alpha} \cap C_{\gamma}$ and $\left(v_{\gamma} v_{\beta}\right) \in C_{\beta} \cap C_{\gamma}$. Hence $\left(v_{\gamma} v_{\alpha}\right) \in$ $G(\mathscr{C})$ and $\left(v_{\gamma} v_{\beta}\right) \in G(\mathscr{C})$, so $\left(v_{\alpha} v_{\beta}\right) \in(G(\mathscr{C}))^{2}$.

Corollary. (Mukhopadhyay) A connected graph $G$ has a square root iff $G$ is locally complete and for some complete family $\mathscr{C} G$ contains no edge of Type III with respect to $\mathscr{C}$.

Proof. Necessity is evident from the construction of the 
induced family. Now let the given conditions hold and let $\left(v_{\alpha} v_{\beta}\right) \in G$. If $\left(v_{\alpha} v_{\beta}\right) \in C_{\alpha} \cap C_{\beta}$ then (1) and (2) of Theorem 4 hold with $\gamma=\alpha$ or $\beta$. If $\left(v_{\alpha} v_{\beta}\right) \notin\left(C_{\alpha} \cup C_{\beta}\right)$, then for some $\gamma(\neq \alpha, \beta) \quad\left(v_{\alpha} v_{\beta}\right) \in C_{\gamma}$. Thus $\left(v_{\alpha} v_{\gamma}\right) \in C_{\gamma}$ and $\left(v_{\beta} v_{r}\right) \in C_{\gamma}$. Since $G$ has no edge of Type III with respect to $\mathscr{C},\left(v_{\alpha} v_{\gamma}\right) \in C_{\alpha}$ and $\left(v_{\beta} v_{\gamma}\right) \in C_{\beta}$. Therefore (1) and (2) of Theorem 4 hold, and $(G(\mathscr{C}))^{2}=G$.

To be used as a test for the existence of a square root, Mukhopadhyay's theorem requires that a complete family $\mathscr{C}$ be found corresponding to which $G$ has no edge of Type III. This is unnecessarily restrictive, as Theorem 4 suggests. In Figure 4, for example, $G_{4}$ has several edges of Type III with respect to $\mathscr{C}$.

A graph $G$ may have more than one square root, and a given square root may be the derived graph corresponding to complete families different from the induced family (again Figure 4). Each square root $H$ of $G$ contains a minimal square root (a concept discussed briefly in [3]), and is contained in a maximal square root $H_{m}$ (i.e., $H \subset H_{m}, H_{m}^{2}=G$, no proper supergraph of $H_{m}$ is a square root). By concentrating on maximal square roots, the theory developed earlier leads to a more efficient square root test (Theorem 5, below).

If $H$ is a nonmaximal square root of $G$, let it be a proper subgraph of the square root $K$. Let $\mathscr{C}^{*}$ be induced by $H$ and $\mathscr{C}^{\prime}$ induced by $K$. Since $G$ has no edge of Type III with respect to either $C^{*}$ or $\mathscr{C}^{\prime}$, by Theorem $3 C_{\alpha}^{*} \subset C_{\alpha}^{\prime}$ for each $\alpha$ and $C_{\beta}^{*} \neq C_{\beta}^{\prime}$ for some $\beta$. This suggests the concept defined next.

Definition 4. The complete family $\mathscr{C}=\left\{C_{\alpha}\right\}$ is called maximal iff for each $\alpha C_{\alpha}$ is a clique.

It is clear that each graph which has a complete family has a maximal complete family and that a given vertex may belong to more than one clique.

THeOREM 5. Let $G$ be locally complete and let $\mathscr{C l}$ denote the set of all maximal complete families for $G$. If $G$ has a square root, then some member of the set $\{G(\mathscr{C}): \mathscr{C} \in \mathscr{M}\}$ is a square root.

Proof. Let $H^{2}=G$ and let $\mathscr{C}^{*}$ be its induced family. For each $\alpha$, let $C_{\alpha}$ be a clique containing $C_{\alpha}^{*}$. Then $\mathscr{C} \in \mathscr{H}, H \subset G(\mathscr{C})$ and $G(\mathscr{C})$ is a square root.

EXAMPLE. Consider the graph shown in Figure 4. First, tabulate the cliques containing each vertex, and note that $G_{4}$ is 


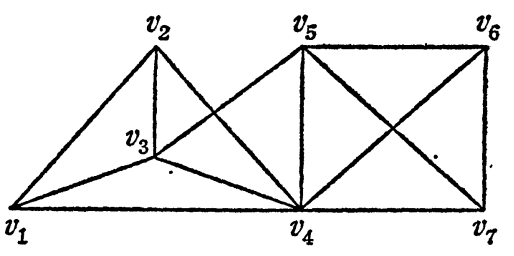

$G_{4}$

$1[1,2,3,4]$

$2[1,2,3,4]$

$3[1,2,3,4] \quad[3,4,5]$

$4[1,2,3,4] \quad[3,4,5]$

$5[3,4,5]$

$6[4,5,6,7]$

$7[4,5,6,7]$

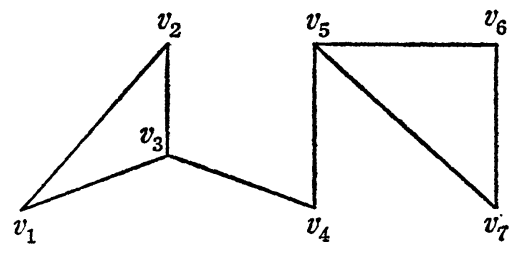

$G_{4}(\mathscr{C})=H$

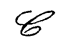

$\mathscr{C}^{*}$

$[1,2,3,4] \quad[1,2,3]$

$[1,2,3,4] \quad[1,2,3]$

$[1,2,3,4] \quad[1,2,3,4]$

$[3,4,5] \quad[3,4,5]$

$[4,5,6,7] \quad[4,5,6,7]$

$[4,5,6,7] \quad[5,6,7]$

$[4,5,6,7] \quad[5,6,7]$

FIGURE 4

locally complete. Among its maximal complete families, some may be eliminated from consideration fairly quickly because the derived graphs are not connected (see Theorem 2 and its corollary). For example, the choice $C_{1}=C_{2}=C_{3}=C_{4}=[1,2,3,4]$ is not admissible. By Theorem 4 , the following complete family $\mathscr{C}$ is admissible:

$$
C_{1}=C_{2}=C_{3}=[1,2,3,4], C_{4}=[2,4,5], C_{5}=C_{6}=C_{7}=[4,5,6,7] \text {. }
$$

The derived graph $G_{4}(\mathscr{C})$ is shown in Figure 4 and it is easily seen to be a square root of $G_{4}$. The induced family $\mathscr{C}^{*}$ is also shown, and it clearly is not maximal even though $G_{4}(\mathscr{C})$ is maximal.

It would be of interest to modify the concepts introduced in this paper for application to directed graphs and to the square roots of digraphs [1].

\section{REFERENCES}

1. D. P. Geller, The square root of a digraph, J. Combinatorial Theory, 5 (1968), 320-321.

2. F. Harary, Graph Theory, Addison-Wesley, Reading, Mass., 1969.

3. A. Mukhopadhyay, The square root of a graph, J. Combinatorial Theory, 2 (1967), 290-295.

Received January 31, 1972.

EMORY UNIVERSITY 


\section{PACIFIC JOURNAL OF MATHEMATICS}

\section{EDITORS}

D. Gilbarg and J. Milgram

Stanford University

Stanford, California 94305

R. A. Beaumont

University of Washington

Seattle, Washington 98105
J. DUGUNDJI* Department of Mathematics

University of Southern California Los Angeles, California 90007

RICHARD ARENS

University of California

Los Angeles, California 90024

\section{ASSOCIATE EDITORS}

E. F. BeCKenbach

B. H. NeUMaNN

F. WOLF

K. Yoshida

\section{SUPPORTING INSTITUTIONS}

UNIVERSITY OF BRITISH COLUMBIA

UNIVERSITY OF SOUTHERN CALIFORNIA

CALIFORNIA INSTITUTE OF TECHNOLOGY

UNIVERSITY OF CALIFORNIA

MONTANA STATE UNIVERSITY

STANFORD UNIVERSITY

UNIVERSITY OF TOKYO

UNIVERSITY OF NEVADA

UNIVERSITY OF UTAH

NEW MEXICO STATE UNIVERSITY

WASHINGTON STATE UNIVERSITY

OREGON STATE UNIVERSITY

UNIVERSITY OF OREGON

OSAKA UNIVERSITY

UNIVERSITY OF WASHINGTON

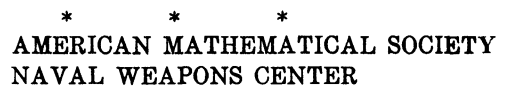

* C. DePrima will replace J. Dugundji until August 1974. 


\section{Pacific Journal of Mathematics}

\section{Vol. 47, No. $1 \quad$ January, 1973}

K. Adachi, Masuo Suzuki and M. Yoshida, Continuation of holomorphic

mappings, with values in a complex Lie group ....................

Michael Aschbacher, A characterization of the unitary and symplectic groups

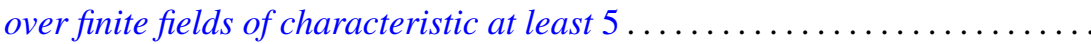

Larry Eugene Bobisud and James Calvert, Energy bounds and virial theorems for abstract wave equations....................................

Christer Borell, A note on an inequality for rearrangements ................

Peter Southcott Bullen and S. N. Mukhopadhyay, Peano derivatives and general

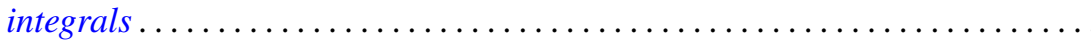

Wendell Dan Curtis, Yu-Lee Lee and Forrest Miller, A class of infinite dimensional subgroups of $\operatorname{Diff}^{r}(X)$ which are Banach Lie groups .........

Paul C. Eklof, The structure of ultraproducts of abelian groups ...............

William Alan Feldman, Axioms of countability and the algebra $C(X) \ldots \ldots \ldots$

Jack Tilden Goodykoontz, Jr., Aposyndetic properties of hyperspaces...........

George Grätzer and J. Płonka, On the number of polynomials of an idempotent algebra. II ...........................................

Alan Trinler Huckleberry, The weak envelope of holomorphy for algebras of

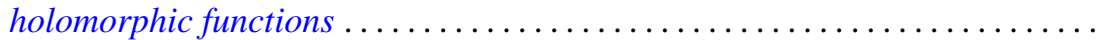

John Joseph Hutchinson and Julius Martin Zelmanowitz, Subdirect sum decompositions of endomorphism rings . . . . . . . . . . . . . . . .

Gary Douglas Jones, An asymptotic property of solutions of

$y^{\prime \prime \prime}+p y^{\prime}+q y=0$.

Howard E. Lacey, On the classification of Lindenstrauss spaces .

Charles Dwight Lahr, Approximate identities for convolution measure algebras.

George William Luna, Subdifferentials of convex functions on Banach

spaces.

Nelson Groh Markley, Locally circular minimal sets. .

Robert Wilmer Miller, Endomorphism rings of finitely generated projective modules

Donald Steven Passman, On the semisimplicity of group rings of linear

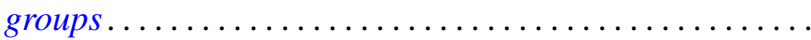

Bennie Jake Pearson, Dendritic compactifications of certain dendritic spaces.

Ryōtarō Satō, Abel-ergodic theorems for subsequences ...... .

Henry S. Sharp, Jr., Locally complete graphs. . .

Harris Samuel Shultz, A very weak topology for the Mikusinski field of

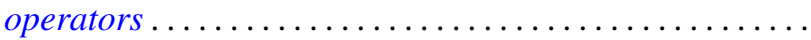

Elena Stroescu, Isometric dilations of contractions on Banach spaces ...

Charles W. Trigg, Versum sequences in the binary system ... . .

William L. Voxman, On the countable union of cellular decompositions of n-manifolds 